\title{
SMALL SCATTER AND NEARLY ISOTHERMAL MASS PROFILES TO FOUR HALF-LIGHT RADII FROM TWO-DIMENSIONAL STELLAR DYNAMICS OF EARLY-TYPE GALAXIES
}

\author{
Michele Cappellari $^{1}$, Aaron J. Romanowsky ${ }^{2,3}$, Jean P. Brodie ${ }^{2}$, Duncan A. Forbes ${ }^{4}$, Jay Strader $^{5}$, Caroline Foster $^{6}$, \\ Sreeja S. Kartha ${ }^{4}$, Nicola Pastorello ${ }^{4}$, Vincenzo Pota ${ }^{2,4}$, Lee R. Spitler ${ }^{6,7}$, Christopher Usher ${ }^{4}$, and Jacob A. Arnold ${ }^{2}$ \\ ${ }^{1}$ Sub-department of Astrophysics, Department of Physics, University of Oxford, Denys Wilkinson Building, Keble Road, Oxford OX1 3RH, UK \\ ${ }^{2}$ University of California Observatories, 1156 High Street, Santa Cruz, CA 95064, USA \\ ${ }^{3}$ Department of Physics and Astronomy, San José State University, One Washington Square, San Jose, CA 95192, USA \\ ${ }^{4}$ Centre for Astrophysics \& Supercomputing, Swinburne University, Hawthorn, VIC 3122, Australia \\ 5 Department of Physics and Astronomy, Michigan State University, East Lansing, MI 48824, USA \\ ${ }^{6}$ Australian Astronomical Observatory, P.O. Box 915, North Ryde, NSW 1670, Australia \\ ${ }^{7}$ Department of Physics and Astronomy, Macquarie University, North Ryde, NSW 2109, Australia \\ Received 2015 January 15; accepted 2015 March 31; published 2015 April 29
}

\begin{abstract}
We study the total mass-density profile for a sample of 14 fast-rotator early-type galaxies (stellar masses $\left.10.2 \lesssim \log M_{*} / M_{\odot} \lesssim 11.7\right)$. We combine observations from the SLUGGS and ATLAS ${ }^{3 \mathrm{D}}$ surveys to map out the stellar kinematics in two dimensions, out to a median radius for the sample of four half-light radii $R_{\mathrm{e}}$ (or $10 \mathrm{kpc}$ ) and a maximum radius of 2.0-6.2 $R_{\mathrm{e}}$ (or 4-21 kpc). We use axisymmetric dynamical models based on the Jeans equations, which allow for a spatially varying anisotropy; employ quite general profiles for the dark halos; and, in particular, do not place any restrictions on the profile slope. This is made possible by the availability of spatially extended two-dimensional kinematics. We find that our relatively simple models provide a remarkably good description of the observed kinematics. The resulting total density profiles are well described by a nearly isothermal power law $\rho_{\text {tot }}(r) \propto r^{-\gamma}$ from $R_{\mathrm{e}} / 10$ to at least $4 R_{\mathrm{e}}$, the largest average deviation being $11 \%$. The average logarithmic slope is $\langle\gamma\rangle=2.19 \pm 0.03$ with observed rms scatter of just $\sigma_{\gamma}=0.11$. This scatter out to large radii, where dark matter dominates, is as small as previously reported by lensing studies around $r \approx R_{\mathrm{e}} / 2$, where the stars dominate. Our bulge-halo conspiracy places much tighter constraints on galaxy formation models. It illustrates the power of two-dimensional stellar kinematics observations at large radii. It is now important to test the generality of our results for different galaxy types and larger samples.
\end{abstract}

Key words: galaxies: elliptical and lenticular, cD - galaxies: formation - galaxies: kinematics and dynamics galaxies: structure

\section{INTRODUCTION}

One of the pillars on which our entire paradigm of galaxy formation rests is the fact that dark matter of an unknown nature dominates the mass budget of the universe (Blumenthal et al. 1984). Without dark matter, the primordial gas would not be able to collapse quickly enough within the center of dark matter halos to form the galaxies we observe (White \& Rees 1978).

Total mass-density profiles of spiral galaxies were obtained decades ago, given the simple geometry of their spiral disks and the availability of ionized (Rubin et al. 1980) and neutral gas (Bosma 1978), which provide a kinematical tracer easy to measure and model (see the review by Courteau et al. 2014). The measurements indicated flat circular rotation curves and consequently nearly isothermal $\rho_{\text {tot }} \propto r^{-2}$ profiles.

The situation is very different for early-type galaxies (ETGs, namely, ellipticals and lenticulars), which, by definition, lack well-defined spiral disks and are cold-gas poor. For ETGs, one usually has to rely on expensive observations of the stellar kinematics and more complex dynamical models. This has restricted most studies to radii not much larger than the halflight radius $R_{\mathrm{e}}$.

A general consensus has emerged for the mass distribution of the ETGs inner parts. Long-slit observations of two different samples of $\approx 20$ ETGs revealed rotation curves to be nearly flat with nearly isothermal mass profiles, as in spiral galaxies, within the median radius $r \approx 2 R_{\mathrm{e}}$ sampled by the kinematics
(Gerhard et al. 2001; Thomas et al. 2011). This agrees with strong gravitational lensing studies finding nearly isothermal slopes, with small scatter, for the total galaxy density profile of 73 ETGs at a typical radius of $r \approx R_{\mathrm{e}} / 2$ (Auger et al. 2010). These central slopes are similar to those measured for group/ cluster-scale profiles using X-ray modeling (e.g., Humphrey \& Buote 2010) or stacked weak-lensing technique (e.g., Gavazzi et al. 2007).

The largest detailed study of dark matter in galaxy centers was based on the integral-field observations of the volumelimited ATLAS $^{3 \mathrm{D}}$ sample of 260 ETGs (Cappellari et al. 2011). It inferred a median dark matter fraction of just $f_{\mathrm{DM}}\left(R_{\mathrm{e}}\right)=13 \%$, within a sphere of radius $R_{\mathrm{e}}$, over the full sample (Cappellari et al. 2013a). This shows that studies restricted to the central regions of ETGs mainly measure the stellar mass distribution.

To explore the region where dark matter dominates, one needs to reach at least $\sim 4 R_{\mathrm{e}}$. Existing studies at these radii targeted one galaxy at a time. They used observations of extended H I disks (Weijmans et al. 2008), deep stellar kinematics at a few sparse locations (Weijmans et al. 2009; Forestell \& Gebhardt 2010; Murphy et al. 2011), globular cluster kinematics (Napolitano et al. 2014), and planetary nebulae (Romanowsky et al. 2003; de Lorenzi et al. 2008, 2009; Das et al. 2011; Napolitano et al. 2011; Morganti et al. 2013).

The fact that galaxies were modeled using different techniques or kinematic tracers, and for a mix of ETGs with 
Table 1

Sample of Fast-rotator Early-type Galaxies and Measured Parameters

\begin{tabular}{|c|c|c|c|c|c|c|c|c|c|c|c|}
\hline $\begin{array}{l}\text { Name } \\
\text { (1) }\end{array}$ & $\begin{array}{c}D \\
(\mathrm{Mpc}) \\
(2)\end{array}$ & $\begin{array}{c}\log R_{\mathrm{e}} \\
\left({ }^{\prime \prime}\right) \\
(3)\end{array}$ & $\begin{array}{c}R_{\max } \\
(\mathrm{kpc}) \\
(4)\end{array}$ & $\begin{array}{c}R_{\max } / R_{\mathrm{e}} \\
(5)\end{array}$ & $\begin{array}{c}\langle\gamma\rangle_{r} \\
\left(0.1-1 R_{\mathrm{e}}\right) \\
(6)\end{array}$ & $\begin{array}{c}\langle\gamma\rangle_{r} \\
\left(1-4 R_{\mathrm{e}}\right) \\
(7)\end{array}$ & $\begin{array}{c}\langle\gamma\rangle_{r} \\
\left(0.1-4 R_{\mathrm{e}}\right) \\
(8)\end{array}$ & $\begin{array}{c}\langle|\Delta|\rangle_{r} \\
\left(0.1-4 R_{\mathrm{e}}\right) \\
(9)\end{array}$ & $\begin{array}{c}\text { MGE Reference } \\
(10)\end{array}$ & $\begin{array}{c}\chi_{\mathrm{JAM}}^{2} / \chi_{\text {LOESS }}^{2} \\
(11)\end{array}$ & $\begin{array}{c}\operatorname{Median}\left(\left|\delta V_{\text {rms }}\right|\right) \\
(\%) \\
(12)\end{array}$ \\
\hline NGC 1023 & 11.1 & 1.68 & 11.3 & 4.39 & -2.20 & -2.19 & -2.20 & 0.009 & S09 & 0.98 & 12.3 \\
\hline NGC 2768 & 21.8 & 1.80 & 19.7 & 2.95 & -2.10 & -1.82 & -2.01 & 0.025 & $\mathrm{C} 06$ & 1.02 & 5.1 \\
\hline NGC 2974 & 20.9 & 1.58 & 10.8 & 2.80 & -2.22 & -2.49 & -2.30 & 0.015 & $\mathrm{C} 06$ & 1.10 & 14.9 \\
\hline NGC 3115 & 9.5 & 1.54 & 10.1 & 6.19 & -2.28 & -2.53 & -2.37 & 0.028 & E99 & 1.10 & 10.7 \\
\hline NGC 4278 & 15.6 & 1.50 & 9.0 & 3.76 & -2.19 & -2.45 & -2.29 & 0.028 & $\mathrm{C} 06$ & 1.09 & 7.8 \\
\hline NGC 4473 & 15.3 & 1.43 & 8.4 & 4.18 & -2.12 & -2.29 & -2.18 & 0.015 & $\mathrm{C} 06$ & 1.20 & 6.4 \\
\hline NGC 4494 & 16.6 & 1.69 & 8.0 & 2.04 & -2.18 & -2.52 & -2.26 & 0.045 & S13 & 1.06 & 9.2 \\
\hline NGC 4526 & 16.4 & 1.65 & 18.2 & 5.13 & -2.21 & -2.29 & -2.24 & 0.014 & $\mathrm{C} 06$ & 0.97 & 9.0 \\
\hline NGC 4649 & 17.3 & 1.82 & 21.4 & 3.86 & -2.10 & -2.34 & -2.19 & 0.020 & S13 & 1.05 & 8.9 \\
\hline NGC 4697 & 11.4 & 1.79 & 12.5 & 3.66 & -2.16 & -2.34 & -2.23 & 0.023 & S13 & 0.99 & 7.1 \\
\hline
\end{tabular}

Notes. Column (1): galaxy name. Columns (2)-(3): distance and half-light radius from Cappellari et al. (2011). Columns (4)-(5): maximum radius $R_{\max }$ sampled by the stellar kinematics. Column (6): average logarithmic slope $\langle\gamma\rangle_{r}=\Delta \log \rho_{\text {tot }} / \Delta \log r$ of the total mass profile in the interval $0.1 R_{\mathrm{e}}<r<R_{\mathrm{e}}$. Column (7): as in column 6, but for $R_{\mathrm{e}}<r<\min \left(4 R_{\mathrm{e}}, R_{\max }\right)$. Column (8): as in column 6, but for $0.1 R_{\mathrm{e}}<r<\min \left(4 R_{\mathrm{e}}, R_{\max }\right)$. Column (9): average absolute deviation between $\rho_{\text {tot }}$ and the best-fitting power law (in dex). Column (10): reference for the MGE model: C06 - Cappellari et al. (2006), S09-Scott et al. (2009), S13-Scott et al. (2013), E99-Emsellem et al. (1999). Column (11): quality of fit, where $\chi_{\mathrm{JAM}}$ is measured from the JAM models and $\chi_{\text {LoEsS }}$ from the smoothed data. In both cases, the reference is the original SLUGGS data, excluding values with zero final weight. This ratio approximates $\chi^{2} / \mathrm{DOF}$, but is insensitive to the normalization of the kinematic uncertainties. Column (12): median of the absolute relative deviations $\left|\delta V_{\mathrm{rms}}\right| \equiv\left|V_{\mathrm{rms}} / V_{\mathrm{rms}}^{\mathrm{LOESS}}-1\right|$ between the original and the smoothed SLUGGS $V_{\mathrm{rms}}$.

axisymmetric and triaxial shapes, resulted in a still unclear picture of the global trends. Tentative conclusions suggest a trend with massive ETGs having nearly isothermal total mass profiles and flat circular velocities (see the review by Gerhard et al. 2013), while lower-mass ones have more slowly rising dark matter profiles and correspondingly falling circular velocity profiles (also Romanowsky et al. 2003).

The situation is changing with the availability of extended stellar kinematics for significant samples of ETGs (Brodie et al. 2014; Raskutti et al. 2014). Here, we present the first detailed and fully homogeneous analysis using large-scale stellar dynamics of a statistically significant sample of 14 ETGs (but see Deason et al. 2012 for less detailed models). The key novelty of this work is the availability of two-dimensional stellar kinematics out to a median radius of $r \approx 4 R_{\mathrm{e}}$ for the sample from the SLUGGS survey (Brodie et al. 2014), which we combine with integral-field kinematics in the central regions $\left(\sim 1 R_{\mathrm{e}}\right)$ from the ATLAS $^{3 \mathrm{D}}$ survey (Cappellari et al. 2011).

\section{SAMPLE AND DATA}

\subsection{Axisymmetric Sample Selection}

We study a subsample of the SLUGGS ETG sample, with kinematics from Arnold et al. (2014). We want a homogeneous set of galaxies, and we use an axisymmetric dynamical modeling method. For this reason, we only select ETGs classified as fast rotators in Emsellem et al. (2011). We add the fast rotator NGC 3115, without ATLAS ${ }^{3 \mathrm{D}}$ kinematics. Our sample of 14 galaxies is given in Table 1 (stellar masses $10.2 \lesssim \log M_{*} / M_{\odot} \lesssim 11.7$ from Capellari et al. 2013a).

Fast rotators were shown by Krajnović et al. (2011) to have kinematical axes, within about $1 R_{\mathrm{e}}$, that are extremely well aligned with the photometric major axes measured at the much larger radii $\sim 3 R_{\mathrm{e}}$ sampled by the SLUGGS kinematics. This alignment, for the statistically significant ATLAS $^{3 \mathrm{D}}$ sample, shows that fast-rotator ETGs, as a class, must be axisymmetric out to the region sampled by our models. Significant deviations from axisymmetry would produce a broad distribution of kinematical misalignment that is clearly ruled out by the ATLAS $^{3 \mathrm{D}}$ data. The SLUGGS kinematics of fast rotators is also generally consistent with axisymmetry and shows small kinematical misalignment.

Some genuine deviations from a bisymmetric velocity field do exist in the SLUGGS data. Notable examples are NGC 4494 in the maps of Arnold et al. (2014) and NGC 4473 in Foster et al. (2013). However, these deviations are more likely due to unrelaxed substructure in the stellar halo, than to nonaxisymmetry in the relaxed stellar distribution. In fact, the tight alignment between ATLAS ${ }^{3 \mathrm{D}}$ stellar kinematics and photometry, as well as the complete lack of a photometric twist, is extremely unlikely in a triaxial configuration (e.g., Binney 1985). In this work, we attribute deviations from the axisymmetric assumption to either substructure or systematic problems in the data and simply try to remove their effects from our models.

\subsection{Photometry and Mass Models}

We use the Multi-Gaussian Expansion (MGE; Emsellem et al. 1994; Cappellari 2002) to parameterize both stellar and dark halo distributions. The MGEs of eight galaxies come from Cappellari et al. (2006) and one from Scott et al. (2009). They include deep $I$-band photometry out to 5-10R $R_{\mathrm{e}}$. Three SDSS $r$ band MGEs come from Scott et al. (2013). The $V$-band MGE for NGC 3115 comes from Emsellem et al. (1999). The MGE for NGC 4111 was redone on the $i$-band SDSS image using the Python version ${ }^{8}$ of the MGE-fitting method of Cappellari (2002) to avoid the $r$-band saturation (see Scott et al. 2013, supplementary material).

\footnotetext{
Available from http://purl.org/cappellari/software.
} 

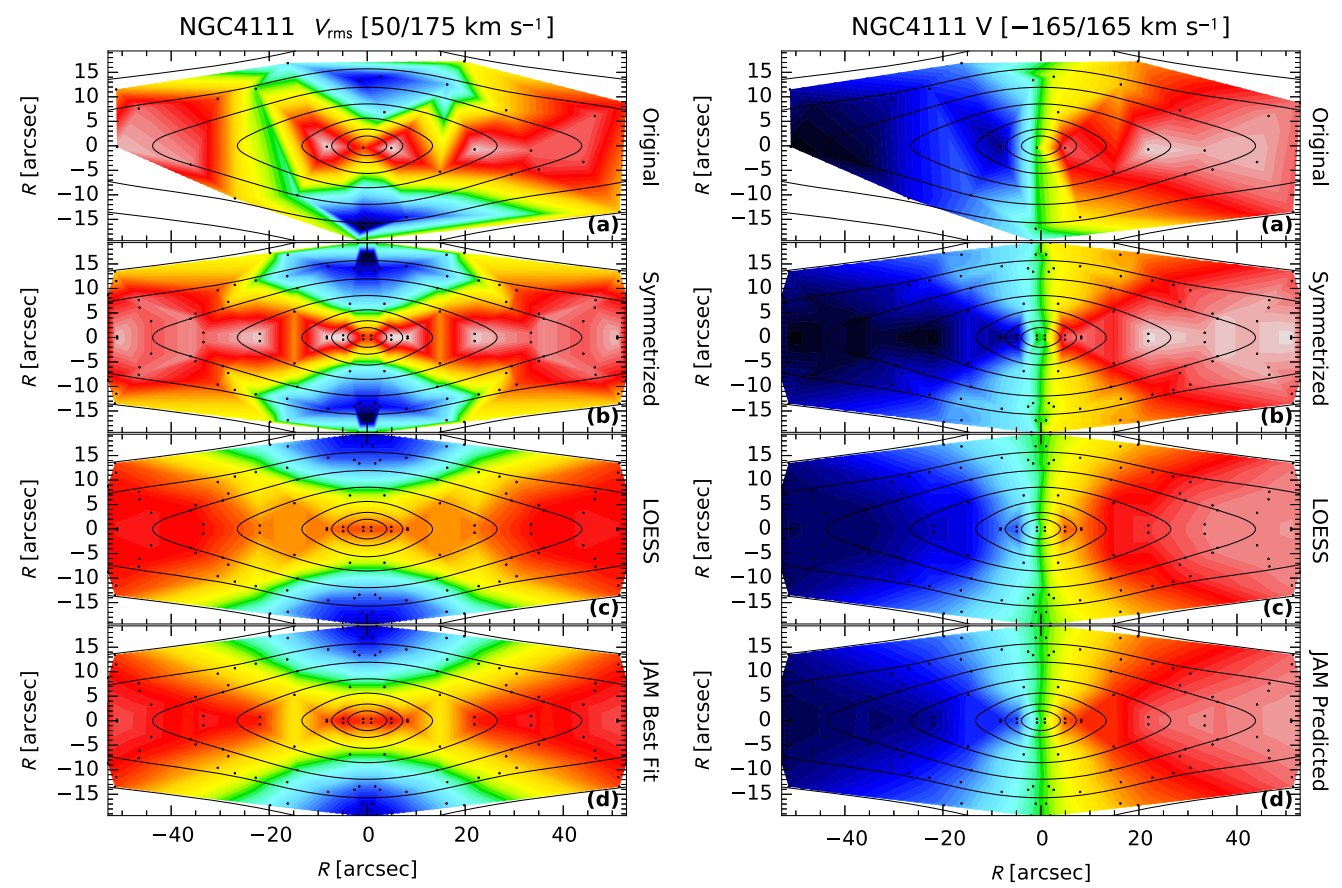

Figure 1. Symmetrization and cleaning of SLUGGS data. The left panels show the stellar $V_{\mathrm{rms}} \equiv \sqrt{V^{2}+\sigma^{2}}$, while the right panels show the mean stellar velocity $V$. (a) Original linearly interpolated data. (b) Bisymmetrized data. (c) Bisymmetrized and LOESS-smoothed data. Note the good prediction, in the central parts, of the actual SAURON kinematics in Figure 2. (d) Best-fitting JAM model. The right panel shows the JAM prediction for the mean velocity using the same model parameters as in the left panel, adopting for all Gaussians $\sigma_{\phi}=\sigma_{R}$ and $\kappa=1.20$. We tried different $\sigma_{\phi} / \sigma_{R}$ ratios ${ }^{10}$ but found the best fits for $\sigma_{\phi} \approx \sigma_{R}$ (see Cappellari 2008 for definitions).

\subsection{Stellar Kinematics}

The kinematics ${ }^{9}$ for the inner $\left(\sim 1 R_{\mathrm{e}}\right)$ region of our galaxies were observed with the SAURON integral-field spectrograph and were homogeneously extracted for the ATLAS 3D survey as described in Cappellari et al. (2011). For 10 of the 14 galaxies, the observations taken as part of the SAURON survey were already presented in Emsellem et al. (2004). The kinematics for the outer regions were observed with the Keck/ DEIMOS multi-slit spectrograph by the SLUGGS survey. They were presented and described in Arnold et al. (2014).

Briefly, in both cases, the kinematics were extracted in pixel space using the pPXF method (Cappellari \& Emsellem 2004). The ATLAS ${ }^{3 \mathrm{D}}$ data covered the $\mathrm{H} \beta$ and $\mathrm{Mg} b$ spectral region (4800-5380 $\AA$ ) and were extracted using the full MILES stellar library (Sánchez-Blázquez et al. 2006) as templates, while the SLUGGS data covered the Ca II triplet spectral region (8480-8750 $\AA$ ) and were extracted using the stellar library of Cenarro et al. (2001).

\section{METHODS}

\subsection{Symmetrization and Outlier Removal}

A key step of this analysis is the removal of potential outliers from the SLUGGS kinematics. These may have significant effects on the modeling results, owing to the relatively sparse sampling of the kinematics at large radii.

When fitting kinematics to models, one can enforce symmetry during the kinematic extraction (e.g., Cappellari \& Emsellem 2004). We adopt here a conceptually similar idea, which is easier to apply to irregularly sampled or discrete kinematics (see also Morganti et al. 2013).

\footnotetext{
Available from http://purl.org/atlas3d.
}

At every observed location $\left(x_{j}, y_{j}\right)$ on the sky, where the $x$ axis coincides with the kinematic major axis $\mathrm{PA}_{\text {kin }}$ (taken from Krajnović et al. 2011), we calculate the $\mathrm{rms}$ velocity $V_{\mathrm{rms}, j}^{2} \equiv V_{j}^{2}+\sigma_{j}^{2}$ from the SLUGGS data, where $V_{j}$ is the mean stellar velocity and $\sigma_{j}$ is the velocity dispersion. We then generate a new set of bisymmetric positions by replicating the observed values four times as follows:

$$
\left\{\begin{aligned}
x_{j} & \rightarrow\left(x_{j},-x_{j}, x_{j},-x_{j}\right) \\
y_{j} & \rightarrow\left(y_{j}, y_{j},-y_{j},-y_{j}\right) \\
V_{\mathrm{rms}, j} & \rightarrow\left(V_{\mathrm{rms}, j}, V_{\mathrm{rms}, j}, V_{\mathrm{rms}, j}, V_{\mathrm{rms}, j}\right) .
\end{aligned}\right.
$$

The measurement uncertainties are increased by $2 \times$. Given the bisymmetry of the model, the $\chi^{2}$ statistic, describing the quality of the fit, is unchanged. Equation (1) applies to the even velocity moments like $V_{\text {rms }}$ and $\sigma$. While for the odd velocity moments like $V$, the last line is replaced by

$$
V_{j} \rightarrow\left(V_{j},-V_{j}, V_{j},-V_{j}\right)
$$

The resulting kinematics measurements are then smoothed with the Python version of the two-dimensional LOESS algorithm of Cleveland (1979), implemented (see footnote 8) by Cappellari et al. (2013b; see the example in Figure 1).

Comparison between the smoothed/cleaned $V_{\text {rms }}^{\mathrm{LOESS}}$ and the original one suggests the SLUGGS uncertainties are underestimated due to systematics, with typical errors of $\sim 10 \%$ (Table 1). The original uncertainties would indicate non-smooth kinematics, but this fact seems inconsistent with the smooth galaxy images.

In the following models, we do not fit the original kinematics, rather the symmetrized/smoothed one. This has 

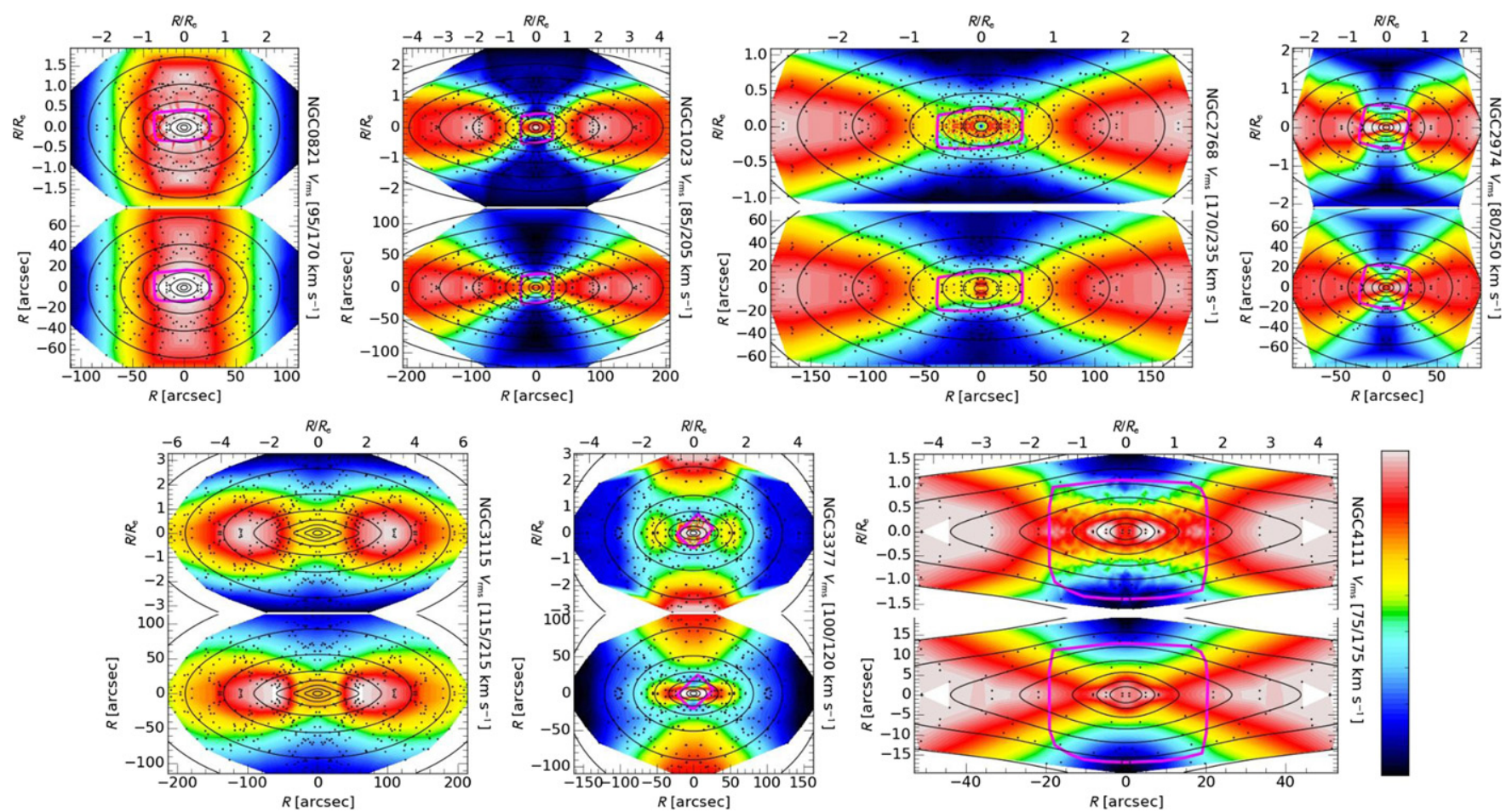

Figure 2. Dynamical models from two-dimensional stellar kinematics. In each panel, the top plot shows the observed stellar $V_{\mathrm{rms}}$, while the bottom plot shows the JAM model prediction. The spatial scale of the top plots is in units of the half-light radius $R_{\mathrm{e}}$. Contours of the MGE surface brightness in 1 mag steps are overlaid. The data include DEIMOS/SLUGGS stellar kinematics at large radii. They were bisymmetrized and LOESS smoothed as described in the text (see Figure 1). The locations sampled by the data are shown as black dots. The SAURON/ATLAS ${ }^{3 \mathrm{D}}$ data, used at smaller radii, are enclosed by the magenta lines.
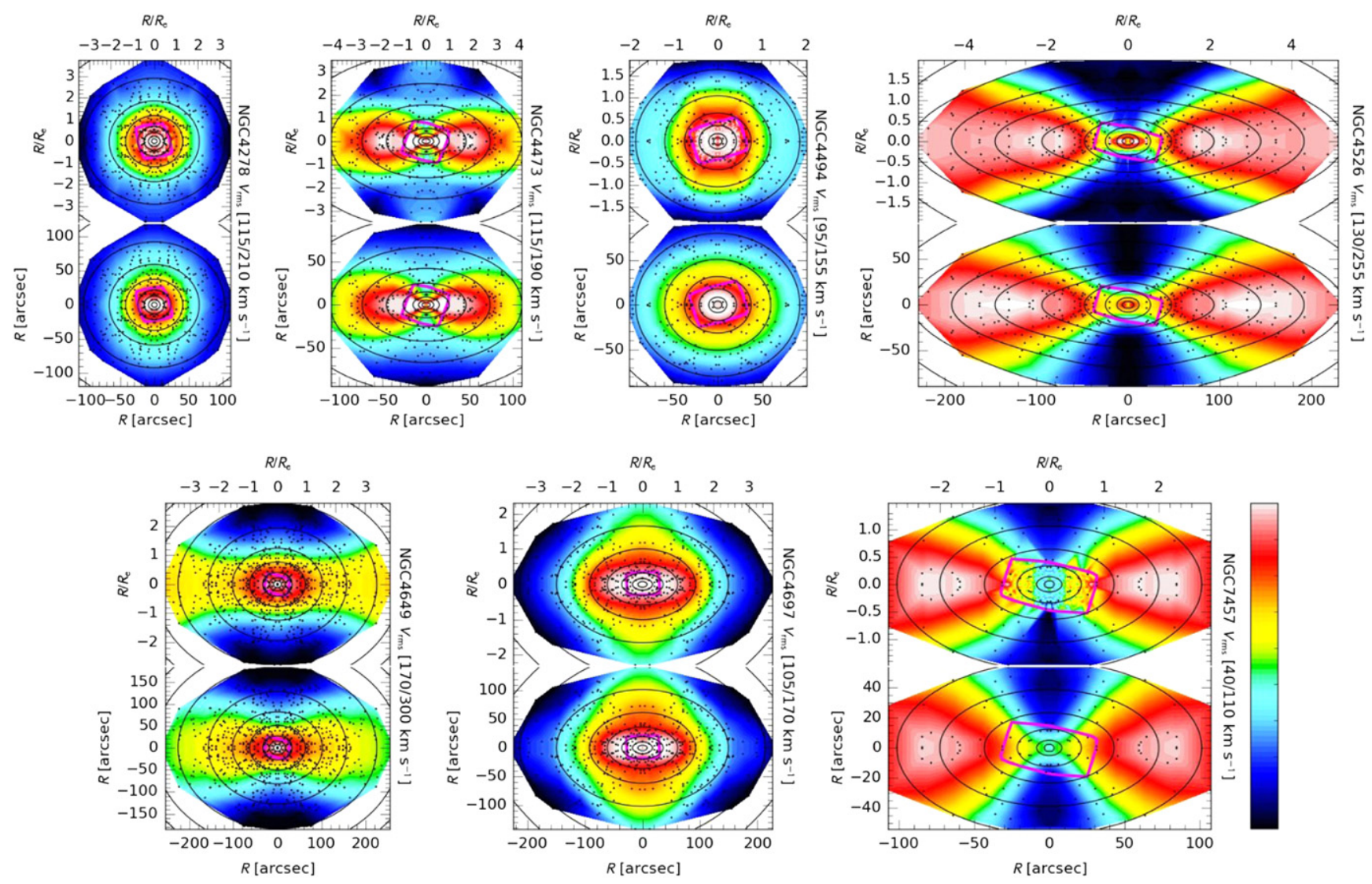

Figure 3. Same as in Figure 2, but for the second half of the galaxy sample. 

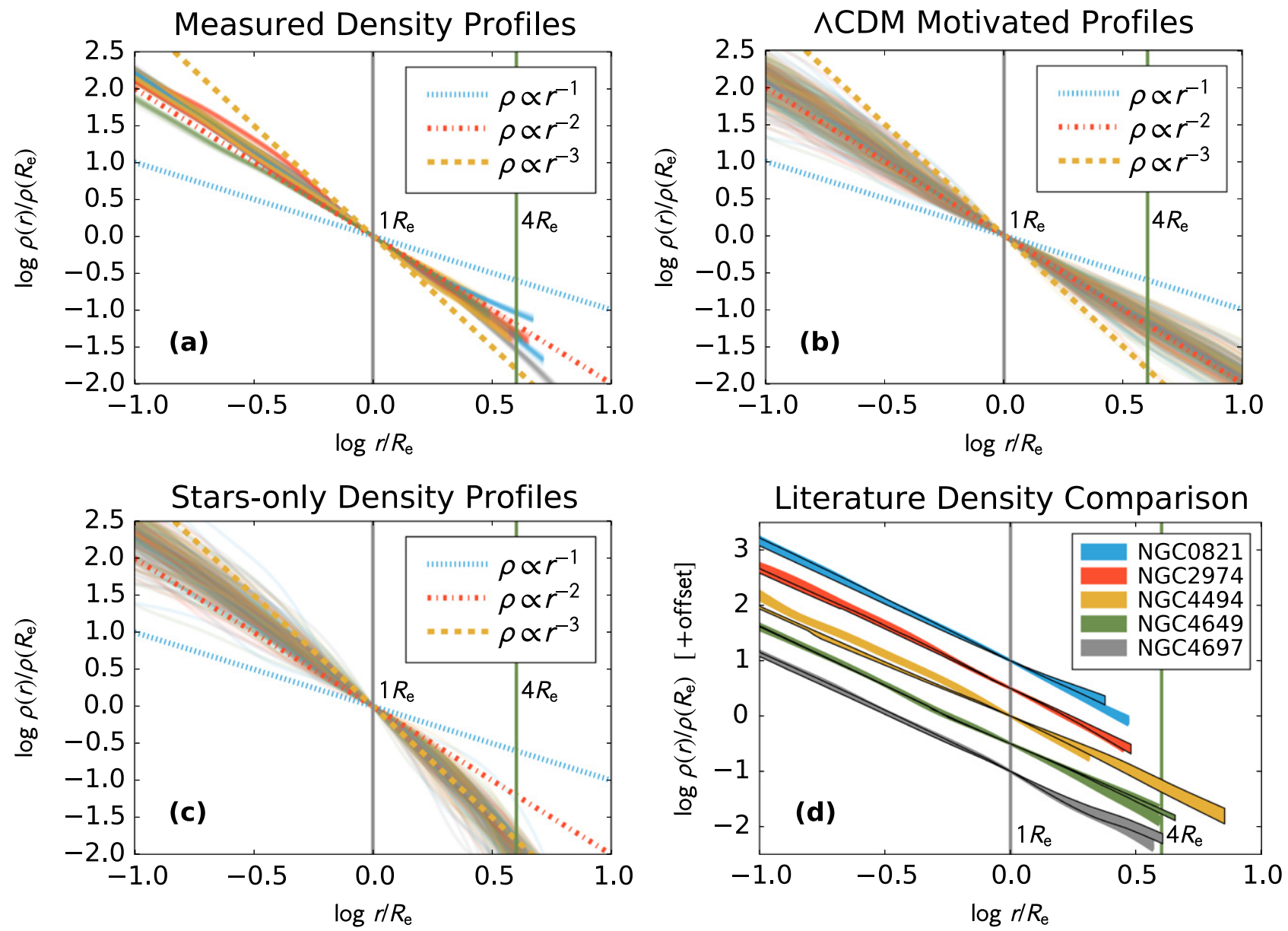

Figure 4. Profiles of the total mass-density distribution. (a) Measured profiles for the 14 modeled galaxies with SLUGGS+ATLAS ${ }^{3 \mathrm{D}}$ data. The profile for each galaxy was plotted for 100 realizations randomly drawn from the posterior distribution of the model parameters to illustrate the random model uncertainties. Three lines with $\rho \propto r^{-1}$ (NFW inner slope), $\rho \propto r^{-2}$ (isothermal), and $\rho \propto r^{-3}$ are also shown. (b) Cosmologically motivated profiles (these are models E from Cappellari et al. 2013a). These were computed by attaching spherical NFW dark halos with masses predicted by the abundance matching technique to the stellar density of the ATLAS galaxies in such a way that the models fit the stellar kinematics. These models naturally predict a nearly isothermal total-mass profile out to $r \gtrsim 10 R_{\mathrm{e}}$. (c) For comparison, the purely stellar profiles of the same ATLAS $^{3 \mathrm{D}}$ galaxies in (b) are shown. (d) Comparison between our density profiles and published ones. The profiles with an outline enclose the allowed range of published profiles, while the colored bands are realizations from the posterior distribution of our model parameters.

the added benefit of making the agreement between data and model easy to visually assess. We need to verify that our models capture the global features of the data and that our results are not driven by a few deviant values. This is important in situations where data systematics may be present, and relying entirely on $\chi^{2}$ statistics may be misleading.

\subsection{Weighting and Matching of the Two Data Sets}

Another issue for the modeling is the fact that the ATLAS ${ }^{3 \mathrm{D}}$ observations consist of many more data points with smaller uncertainties, which completely dominate the $\chi^{2}$ estimate. However, here, we want our dark halo determinations to be especially constrained by the SLUGGS data, which sample the region where the halo dominates. Similarly to Morganti et al. (2013), we increase the size of the ATLAS $^{3 \mathrm{D}}$ kinematic uncertainties so that for a good fit, the two data sets provide an equal contribution to the $\chi^{2}$. We leave the SLUGGS uncertainties unchanged to retain properly normalized confidence levels for our model parameters.

\footnotetext{
${ }^{10}$ This is done by replacing $\mathcal{D}$, which appears in Equation (38) of Cappellari (2008) with $\left[\mathcal{D}+\left(1-c_{k}\right) b_{k} q_{k}^{2} \sigma_{k}^{2} / R^{2}\right]$ (footnote 9 of the arXiv:0806.0042 version of that paper).
}

The SLUGGS $V_{\mathrm{rms}}$ at the SAURON locations tend to be lower than the measured SAURON values. We find a median offset of $11 \%$, which is larger than the $5 \%$ level we consider unavoidable between independent data sets. This offset was noted by Arnold et al. (2014), but its source is unknown. The ATLAS $^{3 \mathrm{D}}$ data agree on average with hundreds of independent determinations from the literature (Figure 8 of Cappellari et al. 2013a), suggesting the SLUGGS data may be offset with respect to the optical literature. Here, we simply multiply the SLUGGS $V_{\text {rms }}$ to fit, for each galaxy, the interpolated SAURON data at the same locations. This is the standard kinematics we fit with our models. However, importantly, we have also run all our models with the SLUGGS data alone and confirmed that the slopes of the total mass profiles agree with those of our standard models.

\subsection{Dynamical Models}

We model the ATLAS ${ }^{3 D}$ and SLUGGS stellar kinematics using the Python version of the axisymmetric Jeans anisotropic modeling (JAM) method (see footnote 8; Cappellari 2008). The approach is the same used in Cappellari et al. (2013a) for 
the $\operatorname{ATLAS}^{3 \mathrm{D}}$ data alone. It employs a Bayesian method with constant (i.e., "ignorant") priors on all parameters.

The key difference between this work and previous stellar dynamical studies of dark halos in ETGs is the fact that we place virtually no constraint on the halo profile parameters. The halo is assumed to be spherical, but it is described by a generalized Navarro et al. (1996) profile (gNFW) with free normalization, inner slope, and break radius:

$$
\rho_{\mathrm{DM}}(r)=\rho_{s}\left(\frac{r}{r_{s}}\right)^{\alpha}\left(\frac{1}{2}+\frac{1}{2} \frac{r}{r_{s}}\right)^{-\alpha-3} .
$$

Our models have seven free parameters. Some are poorly constrained but are not of interest here. They are just "nuisance parameters," marginalized out to derive the total mass profiles studied here. The parameters are (i) the inclination $i$; (ii) the anisotropy $\beta_{z} \equiv 1-\sigma_{z}^{2} / \sigma_{R}^{2}$, with $\sigma_{z}$ and $\sigma_{R}$ the stellar dispersion in cylindrical coordinates, for the MGE Gaussians with $\sigma_{j}<R_{\mathrm{e}}$; (iii) the anisotropy for the remaining Gaussians at larger radii; (iv) the stellar $(M / L)_{\text {stars }}$; (v) the break radius of the dark halo, constrained to be $10<r_{s}<50 \mathrm{kpc}$; (vi) the halo density $\rho_{s}$ at $r_{s}$; and (vii) the dark halo slope $\alpha$ for $r \ll r_{s}$.

\section{RESULTS}

\subsection{The Models Describe the Data Well}

The first result is the simple fact that the models provide a good description of the stellar kinematics of all the modeled galaxies over the full field (Figures 2, 3 and Table 1). This was not expected. It would have been natural if we had employed, e.g., the more general orbit-superposition method (e.g., Cappellari et al. 2006), which is fully described by thousands of parameters. However, our models have just six nonlinear parameters and one scaling factor $(M / L)_{\text {stars }}$.

Moreover, the fits look similarly good even assuming a power-law halo and a constant-anisotropy stellar body (four nonlinear parameters and one scaling factor). The fact that four parameters are able to consistently describe all features of the two-dimensional maps for the full set of 14 galaxies indicates that (i) the (cleaned) SLUGGS data are reliable and (ii) the model assumptions provide a good description of the dynamics and mass distribution of the real galaxies.

An alternative interpretation for the good fits would be that the anisotropy and dark matter variations and data systematics conspire to mimic the simple orbital structure and mass distribution assumed by the models. This would be a realistic possibility when studying a single galaxy, given the non-full generality of the JAM models, but such a conspiracy is unlikely for such a large and diverse set of galaxies.

\subsection{Isothermal Profiles with Small Scatter to $4 R_{\mathrm{e}}$}

The second and main result of this work is that all 14 modeled fast-rotator ETGs have a nearly isothermal $\rho_{\text {tot }} \propto r^{-2}$ total density distribution from $R_{\mathrm{e}} / 10$ out to the median radius of $4 R_{\mathrm{e}}$ sampled by this study (Figure $4(\mathrm{a})$ ). The total massdensity profiles ${ }^{11}$, marginalized over all nuisance parameters,

\footnotetext{
${ }^{11}$ Computed from the axisymmetric MGEs as

$\rho_{\mathrm{tot}}(r)=\sum_{j=1}^{M} \frac{M_{j} \exp \left[-r^{2} /\left(2 \sigma_{j}^{2}\right)\right] \operatorname{erf}\left[r \sqrt{1-q_{j}^{2}} /\left(q_{j} \sigma_{j} \sqrt{2}\right)\right]}{4 \pi \sigma_{j}^{2} r \sqrt{1-q_{j}^{2}}}$.
}

are tightly constrained by the data. In the whole range $0.1 R_{\mathrm{e}}$ $<r<4 R_{\mathrm{e}}$, the profiles are well described by a power law $\rho_{\text {tot }} \propto r^{-\gamma}$ with the largest average deviation of $11 \%$. The corresponding average logarithmic slope is $\langle\gamma\rangle=2.19 \pm 0.03$ for the sample, with an rms scatter of just $\sigma_{\gamma}=0.11$. We do not detect any significant correlation of the slope with $R_{\mathrm{e}}$, stellar mass, or stellar velocity dispersion. For $0.1 R_{\mathrm{e}}<r<R_{\mathrm{e}}$, the average slope is $\langle\gamma\rangle=2.15 \pm 0.03$ with $\sigma_{\gamma}=0.10$, while for $R_{\mathrm{e}}<r<4 R_{\mathrm{e}}$, we find $\langle\gamma\rangle=2.27 \pm 0.06$ with $\sigma_{\gamma}=0.23$.

Our inner-profile slope and scatter are in excellent agreement with the values $\langle\gamma\rangle=2.08 \pm 0.03$, with $\sigma_{\gamma}=0.16$ found around $r \approx R_{\mathrm{e}} / 2$ using strong lensing (Auger et al. 2010).

Figure 4(b) shows that the observed trend is consistent with what one would predict for the whole ATLAS ${ }^{3 \mathrm{D}}$ sample for cosmologically motivated uncontracted Navarro-Frenk-White (NFW) halos. In Figure 4(c), the stellar profiles are very different from the total ones at the radii we sample: they have slopes $\rho_{\text {stars }} \propto r^{-2}$ around $r \approx R_{\mathrm{e}} / 2$ (Figure 2 of Cappellari et al. 2013a) but fall off more steeply than $\rho_{\text {stars }} \propto r^{-3}$ around $r \approx 4 R_{\mathrm{e}}$.

Figure 4(d) compares our total profiles with published ones for NGC 0821, NGC 2974, NGC 4494, NGC 4649, and NGC 4697 (from Forestell \& Gebhardt 2010; Weijmans et al. 2008; Morganti et al. 2013; Das et al. 2011; de Lorenzi et al. 2008 , respectively). The $\rho_{\text {tot }}(r)$ was derived from the circular velocities $v_{c}(r)$, assuming spherical symmetry. In four out of five cases, the agreement is excellent, with our statistical uncertainties overlapping the published profiles over the full radial range. The tight agreement for NGC 2974 is noteworthy, where the $v_{c}(r)$ was directly measured from a regular $\mathrm{H}$ I disk. We believe the disagreement for NGC 4494 may be due to the inclusion of the strong asymmetry in the SLUGGS data at $r>100^{\prime \prime}$ in Morganti et al. (2013) models. We excluded those data from our fits, but including them would improve the agreement.

\section{CONCLUSIONS}

We combine the integral-field stellar kinematics from the ATLAS $^{3 \mathrm{D}}$ survey, within $\sim 1 R_{\mathrm{e}}$, with the two-dimensional stellar kinematics from the SLUGGS survey, out to a median radius of about $4 R_{\mathrm{e}}$ and a maximum radius of $2.0-6.2 R_{\mathrm{e}}$, for a sample of fast-rotator ETGs consistent with axisymmetry. We construct the first statistically significant set of detailed axisymmetric dynamical models of the two-dimensional stellar kinematics out to those large radii where dark matter dominates.

We find that the galaxies' dynamics are well represented by a few relatively simple assumptions. The models tightly constrain the total density profiles, which closely approximate the isothermal form $\rho_{\text {tot }} \propto r^{-2}$ from $R_{\mathrm{e}} / 10$ out to the median radius of $4 R_{\mathrm{e}}$ sampled by the data, with remarkably little scatter. The observed total mass distribution is not a generic prediction of $\Lambda \mathrm{CDM}$ and provides constraints on the models (e.g., Remus et al. 2013; Dutton \& Treu 2014).

Our sample highlights the importance of similar studies on larger samples of galaxies to provide a much needed benchmark for galaxy formation models. For this, studies like the present one, using DEIMOS on Keck or MUSE on the Very Large Telescope, can be complemented with models of shallower data, but for much larger samples like MaNGA (Bundy et al. 2015). To be most useful, samples need to 
include both spiral galaxies and ETGs. These should be modeled in a fully homogeneous way by describing the kinematics of both the stars and the gas within the same gravitational potential. Large radio surveys of the neutral $\mathrm{H}$ I gas like Apertif (Verheijen et al. 2008) can complement MaNGA at the largest radii. The future availability of large kinematic data sets indicates a bright future for the systematic investigation of the mass profiles in galaxies.

M.C. acknowledges support from a Royal Society University Research Fellowship. Supported by National Science Foundation grants AST-0909237 and AST-1211995. D.A.F. thanks the ARC for financial support via DP130100388.

\section{REFERENCES}

Arnold, J. A., Romanowsky, A. J., Brodie, J. P., et al. 2014, ApJ, 791, 80 Auger, M. W., Treu, T., Bolton, A. S., et al. 2010, ApJ, 724, 511

Binney, J. 1985, MNRAS, 212, 767

Blumenthal, G. R., Faber, S. M., Primack, J. R., \& Rees, M. J. 1984, Natur, 311,517

Bosma, A. 1978, PhD thesis, Groningen Univ.

Brodie, J. P., Romanowsky, A. J., Strader, J., et al. 2014, ApJ, 796, 52

Bundy, K., Bershady, M. A., Law, D. R., et al. 2015, ApJ, 798, 7

Cappellari, M. 2002, MNRAS, 333, 400

Cappellari, M. 2008, MNRAS, 390, 71

Cappellari, M., Bacon, R., Bureau, M., et al. 2006, MNRAS, 366, 1126

Cappellari, M., \& Emsellem, E. 2004, PASP, 116, 138

Cappellari, M., Emsellem, E., Krajnović, D., et al. 2011, MNRAS, 413, 813

Cappellari, M., McDermid, R. M., Alatalo, K., et al. 2013b, MNRAS, 432, 1862

Cappellari, M., Scott, N., Alatalo, K., et al. 2013a, MNRAS, 432, 1709

Cenarro, A. J., Cardiel, N., Gorgas, J., et al. 2001, MNRAS, 326, 959

Cleveland, W. 1979, J. Am. Stat. Assoc., 74, 829

Courteau, S., Cappellari, M., de Jong, R. S., et al. 2014, RvMP, 86, 47

Das, P., Gerhard, O., Mendez, R. H., Teodorescu, A. M., \& de Lorenzi, F. 2011, MNRAS, 415, 1244

de Lorenzi, F., Gerhard, O., Coccato, L., et al. 2009, MNRAS, 395, 76

de Lorenzi, F., Gerhard, O., Saglia, R. P., et al. 2008, MNRAS, 385, 1729
Deason, A. J., Belokurov, V., Evans, N. W., \& McCarthy, I. G. 2012, ApJ, 748,2

Dutton, A. A., \& Treu, T. 2014, MNRAS, 438, 3594

Emsellem, E., Cappellari, M., Krajnović, D., et al. 2011, MNRAS, 414, 888

Emsellem, E., Cappellari, M., Peletier, R. F., et al. 2004, MNRAS, 352, 721

Emsellem, E., Dejonghe, H., \& Bacon, R. 1999, MNRAS, 303, 495

Emsellem, E., Monnet, G., \& Bacon, R. 1994, A\&A, 285, 723

Forestell, A. D., \& Gebhardt, K. 2010, ApJ, 716, 370

Foster, C., Arnold, J. A., Forbes, D. A., et al. 2013, MNRAS, 435, 3587

Gavazzi, R., Treu, T., Rhodes, J. D., et al. 2007, ApJ, 667, 176

Gerhard, O. 2013, in IAU Symp. 295, The Intriguing Life of Massive Galaxies ed. D. Thomas, A. Pasquali, \& I. Ferreras (Cambridge: Cambridge Univ. Press), 211

Gerhard, O., Kronawitter, A., Saglia, R. P., \& Bender, R. 2001, AJ, 121, 1936

Humphrey, P. J., \& Buote, D. A. 2010, MNRAS, 403, 2143

Krajnović, D., Emsellem, E., Cappellari, M., et al. 2011, MNRAS, 414, 2923

Morganti, L., Gerhard, O., Coccato, L., Martinez-Valpuesta, I., \& Arnaboldi, M. 2013, MNRAS, 431, 3570

Murphy, J. D., Gebhardt, K., \& Adams, J. J. 2011, ApJ, 729, 129

Napolitano, N. R., Pota, V., Romanowsky, A. J., et al. 2014, MNRAS, 439, 659

Napolitano, N. R., Romanowsky, A. J., Capaccioli, M., et al. 2011, MNRAS, 411, 2035

Navarro, J. F., Frenk, C. S., \& White, S. D. M. 1996, ApJ, 462, 563

Raskutti, S., Greene, J. E., \& Murphy, J. D. 2014, ApJ, 786, 23

Remus, R.-S., Burkert, A., Dolag, K., et al. 2013, ApJ, 766, 71

Romanowsky, A. J., Douglas, N. G., Arnaboldi, M., et al. 2003, Sci, 301, 1696

Rubin, V. C., Ford, W. K. J., \& Thonnard, N. 1980, ApJ, 238, 471

Sánchez-Blázquez, P., Peletier, R. F., Jiménez-Vicente, J., et al. 2006, MNRAS, 371, 703

Scott, N., Cappellari, M., Davies, R. L., et al. 2009, MNRAS, 398, 1835

Scott, N., Cappellari, M., Davies, R. L., et al. 2013, MNRAS, 432, 1894

Thomas, J., Saglia, R. P., Bender, R., et al. 2011, MNRAS, 415, 545

Verheijen, M. A. W., Oosterloo, T. A., van Cappellen, W. A., et al. 2008, in AIP Conf. Ser. 1035, The Evolution of Galaxies Through the Neutral Hydrogen Window ed. R. Minchin, \& E. Momjian (Melville, NY: AIP), 265

Weijmans, A.-M., Cappellari, M., Bacon, R., et al. 2009, MNRAS, 398, 561

Weijmans, A.-M., Krajnović, D., van de Ven, G., et al. 2008, MNRAS, 383, 1343

White, S. D. M., \& Rees, M. J. 1978, MNRAS, 183, 341 